\title{
Diabetes mellitus e coração: um continuum de risco Quais os alvos contemporâneos de tratamento e como alcançá-los?
}

\author{
Diabetes mellitus and heart: a continuum of risk \\ Contemporary targets of treatment and how to reach them?
}

Marília B. Gomes

Aline Tiemi K. Silva

Pedro P. M. Spineti*

\section{Resumo}

As doenças cardiovasculares são a maior causa de morbidade e mortalidade em pacientes diabéticos tipos 1 e 2 . Os mecanismos fisiopatológicos propostos para o aumento do risco cardiovascular em pacientes diabéticos baseiam-se em uma associação entre hiperglicemia e alterações metabólicas intracelulares que resultariam em estresse oxidativo, inflamação subclínica crônica e disfunção endotelial. Os fatores clínicos, como obesidade, dislipidemia e hipertensão arterial, que podem coexistir com o diabetes são de relevante importância e favorecem o aumento do risco cardiovascular inerente à doença. $\mathrm{O}$ propósito desse artigo é descrever a relação contínua entre o controle glicêmico, o estresse oxidativo, os sinais de resistência insulínica, a inflamação subclínica e o risco cardiovascular. Também será abordado o papel das principais comorbidades que contribuem para aumentar o risco cardiovascular desses pacientes. O estabelecimento de alvos clínicos e metabólicos adequados para o paciente diabéti- co será discutido igualmente, dentro do contexto de intervenções terapêuticas medicamentosas e não medicamentosas.

Descritores: Diabetes mellitus; Doenças cardiovasculares; Hipertensão; Glicemia; Dislipidemias; Terapêutica.

\begin{abstract}
Cardiovascular diseases are the most prevalent cause of morbidity and mortality among patients with type 1 or type 2 diabetes. The proposed mechanisms for the increased cardiovascular risk (CV) in this population are poorly understood. It has been suggested that an association between hyperglycemia and intracellular metabolic changes can result in oxidative stress, low-grade inflammation, and endothelial dysfunction. Some clinical factors that may coexist with diabetes such as obesity, dyslipidemia, and hypertension are also important and may increase the $\mathrm{CV}$ risk of diabetes. The aim of this article is to discuss


the continuous link between glucose control, oxidative stress, insulin resistance, and subclinical inflammation with $\mathrm{CV}$ risk. We discuss the estabilished treatment goals for the diabetic patient in the context of pharmacological and non-pharmacological therapeutics.

Keywords: Diabetes mellitus; Cardiovascular diseases; Hypertension; Blood glucose; Dyslipidemias; Therapeutics.

\section{Introdução}

O diabetes mellitus é uma doença crônica cuja prevalência está aumentando globalmente, sendo considerada uma epidemia. Em 1985 a Organização Mundial de Saúde (OMS) estimou que existiam então 30 milhões de pessoas com diabetes no mundo. Esse número aumentou para 135 milhões em 1995 e em 2005 chegou a 227 milhões. No ano de 2030 a OMS prevê que existirão 366 milhões de indivíduos com diabetes no mundo. ${ }^{1} \mathrm{O}$ aumento da prevalência do diabetes se deve principalmente ao aumento da prevalência de diabetes mellitus tipo 2 (DM2) e ocorre em países desenvolvidos e em desenvolvimento. ${ }^{1}$ De maneira semelhante ao que ocorre com o DM2, no entanto, a incidência de diabetes mellitus tipo 1 (DM1) também está crescendo mundialmente..$^{2-3}$

Indivíduos com diabetes e com um controle metabólico cronicamente inadequado têm maior risco de evolução para complicações micro e macrovasculares, resultando em uma diminuição significativa da expectativa e da qualidade de vida e onerando a sociedade pelo aumento dos custos diretos, relacionado aos cuidados médicos, e indiretos, como a perda de produtividade, resultado da relativa morbidade e mortalidade prematura da doença. ${ }^{4}$ Os custos diretos e indiretos atribuídos ao diabetes, em 2007, nos Estados Unidos da América (EUA), foram estimados em 174 bilhões de dólares, com um maior custo em relação a complicações crônicas atribuídas ao diabetes do que no cuidado do próprio diabetes. ${ }^{4}$ A Federação Internacional do Diabetes estimou que o gasto com a doença é de 5 a 10\% do orçamento total do sistema de saúde de muitos países. ${ }^{2}$ As doenças cardiovasculares são as causas mais prevalentes de mortalidade e morbidade nos indivíduos com DM1 e DM2. Em 2004, nos EUA, a presença de doenças cardiovasculares (DCV) e acidente vascular cerebral (AVC) foi observada em 68\% e $16 \%$, respectivamente, das mortes relacionadas ao diabetes em pessoas com mais de 65 anos. ${ }^{5}$ Adultos com diabetes apresentam taxas de mortalidade devido à doença coronariana $\mathrm{e}$ AVC duas a quatro vezes maior que aqueles não diabéticos. ${ }^{5}$ Tem sido estabelecido que pacientes com DM2 sem uma história prévia de infarto do miocárdio têm o mesmo risco de doença coronariana (DAC) que não diabéticos com histórico de infarto agudo do miocárdio (IAM). ${ }^{6}$ Isso levou o National Cholesterol Education Program (NCEP) a considerar o diabetes como um risco equivalente de DAC.?

No entanto, ainda existem incertezas quanto ao fato de o risco cardiovascular conferido pelo diabetes ser verdadeiramente equivalente ao de um IAM prévio. ${ }^{8}$ Geralmente, pacientes com diabetes apresentam outras comorbidades associadas, como obesidade, hipertensão arterial sistêmica (HAS) e dislipidemia, que também contribuem para aumentar o risco cardiovascular. ${ }^{5}$ No período de 2005 a 2008, a Associação Americana de Diabetes (ADA) estimou que $67 \%$ dos indivíduos com diabetes com mais de 20 anos apresentavam pressão arterial maior que 140/90 ou estavam usando medicamentos anti-hipertensivos. ${ }^{9}$

Atualmente, existem evidências que ratificam a relação custo-benefício de programas de melhoria do controle glicêmico e dos outros fatores de risco cardiovascular em pacientes com DM $2{ }^{10} \mathrm{e}$ DM1. ${ }^{11}$ A maioria desses pacientes dificilmente atinge as metas estabelecidas pelas diretrizes preconizadas pelas diferentes sociedades médicas. ${ }^{12,13}$

O principal mecanismo que causa a aterosclerose acelerada em pacientes com diabetes e consequentemente o continuum risco entre ambas as doenças ainda não é esclarecido. 
Esta revisão abordará esse controverso tema no contexto do estresse oxidativo, marcadores de resistência insulínica e de inflamação subclínica. Também serão discutidas as metas de controle dos principais alvos terapêuticos para redução do risco cardiovascular dos pacientes com diabetes.

\section{Mecanismos patogenéticos}

\section{Estresse oxidativo}

A hiperglicemia e, consequentemente, o aumento da glicose intracelular induzem a ativação da via dos polióis, a formação de produtos finais de glicação avançada (AGE), o estresse oxidativo, as anormalidades lipídicas, além da ativação da proteína quinase C (PKC). Desenvolve-se estado inflamatório persistente, dano osmótico, alteração da função de proteínas e fatores de transcrição, além de dano ao DNA celular, ${ }^{14}$ que associado ao acometimento na micro e na macrovasculatura representa a base da patogênese das complicações diabéticas. $\mathrm{O}$ estresse oxidativo e a disfunção endotelial são considerados eventos precoces na história natural do diabetes e poderiam ser o denominador comum através do qual fatores ambientais, como hiperglicemia, hipertensão arterial e dislipidemia, atuam. Sabe-se que pacientes expostos precocemente à hiperglicemia desenvolvem uma "memória hiperglicêmica" que os expõe a um maior risco de complicações futuras, sendo esta mediada por espécies reativas de oxigênio (EROs), que são moléculas quimicamente instáveis e que funcionam como mensageiros secundários na regulação da expressão de genes sensíveis ao sinal redox (ex.: gene do NFkB) e na síntese de moléculas fisiologicamente ativas (ex.: mediadores inflamatórios). Outro mecanismo fisiopatológico das complicações macrovasculares do DM inclui a ativação do sistema renina-angiotensina (SRA), que é acompanhado por um estado pró-inflamatório/pró-fibrótico, envolvendo remodelamento vascular e do miocárdico em animais. ${ }^{14}$ Atualmente consideramos que o estresse oxidativo é resultado de um dese- quilíbrio entre a produção de EROs e da defesa antioxidante. Os EROs são neutralizados por um sistema antioxidante que inclui enzimas (superóxido dismutase, glutationa peroxidase, catalase) e sistema não enzimático (glutation, vitaminas A, C e E). Quantitativamente, a albumina e o ácido úrico são os principais antioxidantes extracelulares, sendo a haptoglobina que se liga a hemoglobina livre do plasma outro importante antioxidante. ${ }^{14}$

\section{Epigenética}

Atualmente existem fortes evidências ligando fatores epigenéticos com certas doenças, incluindo o diabetes e DCV. Os fatores epigenéticos, através de diferentes tipos de reações, parecem mediar a relação entre os genes e o meio ambiente, o que resulta em uma ativação ou repressão da transcrição genética. As reações epigenéticas mais importantes que alteram a transcrição genética são a acetilação e a metilação. Essas reações ocorrem principalmente na cauda das histonas que são proteínas que envolvem o DNA.

Recentemente foi demonstrado em células endoteliais da aorta humana que o excesso de EROs que resultam da presença da hiperglicemia podem induzir monometilação da lisina da histona 3, aumentando assim a expressão da subunidade p65 do fator nuclear kappa beta. Essa reação é responsável pela transcrição aumentada da VCAM1 (molécula de adesão celular-vascular-1) e da MCP1 (molécula quimiotática de monócitos-1), que estão relacionadas com as patologias macrovasculares induzidas pela hiperglicemia. Todavia, essa reação persistiu após 6 dias de alcançada a normoglicemia, dando suporte ao conceito de memória metabólica. ${ }^{15}$

\section{Cascata inflamatória, diabetes e aterosclerose}

A aterosclerose é uma doença complexa, multifatorial e sua aceleração em pacientes com diabetes pode ser explicada por várias condições, incluindo hiperglicemia, aumento 
do estresse oxidativo, produtos de glicação avançada (AGE), dislipidemia, desequilíbrio autonômico, hiperinsulinemia, marcadores inflamatórios e variáveis genéticas. ${ }^{14}$ Diabetes, obesidade e resistência insulínica são condições clínicas associadas a um processo inflamatório subclínico caracterizado por aumento de expressão de citocinas produzidas pelo tecido adiposo, principalmete o visceral, macrófagos ativados e outras células. Mediadores inflamatórios, tais como TNF- $\alpha$, interleucina-1 (IL-1), IL-6, leptina, resistina, MCP-1, inibidor do ativador do plasminogénio-1 (PAI-1), proteína C-reativa (PCR), fibrinogênio, angiotensina, visfatina, retinol-binding protein-4 e adiponectina estão envolvidos em vias de sinalização da insulina e perpetuação da resposta inflamatória. ${ }^{14}$ Essas citocinas estão envolvidas no processo inflamatório crônico da parede de vasos, promovendo o acúmulo de lípidos com consequente desenvolvimento de aterosclerose e das doenças cardiovasculares. ${ }^{14}$

\section{Disfunção endotelial}

Sabe-se que o endotélio é um tecido dinâmico que possui ações críticas para a homeostase sistêmica. Entre suas principais funções podemos citar: manutenção da fluidez sanguínea, controle do tônus vascular por meio da secreção de substâncias vasoativas, regulação da proliferação das células musculares lisas vasculares (VSMC), participação na reação inflamatória local e hemostasia sanguínea.

A disfunção endotelial é um evento precoce na história natural do diabetes e aparece como um denominador comum na fisiopatologia das complicações crônicas da doença. A integridade na produção de NO, que evidencia a vasodilatação dependente do endotélio, pode ser avaliada, principalmente, por estímulos fisiológicos, tais como a hiperemia reativa pós-oclusiva e a hiperemia térmica e, ainda, por estímulos farmacológicos, como a resposta vasodilatora após a administração transcutânea de acetilcolina. Por sua vez, a vasodilatação produzida pela administração de nitroprussiato de sódio (NPS) ou derivados (doador de NO) reflete a integridade estrutural do vaso, ou seja, a resposta independente do endotélio. ${ }^{16} \mathrm{~A}$ hiperglicemia resulta em aumento dos EROs, capazes de inativar o óxido nítrico (NO) derivado do endotélio. Adicionalmente, no endotélio de pacientes diabéticos, o óxido nítrico sintase (NOS) pode desviar a produção de NO para gerar O- em condições de deficiência del-arginina ou tetraidropterina. Quando ambos são produzidos, ocorre formação de peroxinitrito (NOO-), causador de dano a estruturas celulares.

\section{Alvos terapêuticos}

Diversas medidas têm sido empregadas na tentativa de reduzir os eventos cardiovasculares nos pacientes com diabetes. Os principais alvos terapêuticos são o controle da glicemia e o controle dos demais fatores de risco cardiovasculares que se encontram frequentemente associados ao diabetes. Entre eles destacam-se a obesidade, a dislipidemia a HAS e o tabagismo.

\section{O papel do controle glicêmico}

Nas décadas recentes, vários ensaios clínicos têm investigado o efeito do tratamento intensivo da hiperglicemia na diminuição do risco cardiovascular no paciente com DM2 $2^{17-20}$ e DM1, ${ }^{21}$ apresentando conflitos nos resultados. As características clínicas das populações estudadas em relação à presença de DCV e à duração do diabetes, assim como o tipo de intervenção realizada e as metas a serem atingidas, explicam parcialmente as diferenças obtidas nos resultados.

No Estudo Prospectivo de Diabetes do Reino Unido (UKPDS), ${ }^{17}$ em pacientes com DM2 recém-diagnosticados, o tratamento intensivo precoce da hiperglicemia nos primeiros 5 anos da doença resultou, a longo prazo, em benefício cardiovascular em comparação ao grupo de pacientes em tratamento convencional. No entanto, o mesmo não foi observado nos outros 3 grandes estudos clínicos realizados em pacientes com diabetes tipo 2. No Veterans Affairs Diabetes Trial (VADT), ${ }^{18}$ com pacientes 
mais idosos e média de 10 anos de diabetes, não houve benefício cardiovascular quando os mesmos foram submetidos a um regime intensivo da hiperglicemia.

Ressaltamos que essa população era composta por $40 \%$ dos pacientes com história prévia de doença cardiovascular. Resultados semelhantes foram obtidos no estudo Action in Diabetes and Vascular Disease: Preterax and Diamicron Modified Release Controlled Evaluation (ADVANCE) Trial, ${ }^{19}$ que teve como objetivo alcançar uma A1c de 6,5\%, através de tratamento intensivo com gliclazida e mais outras drogas. Essa estratégia não reduziu a taxa de eventos macrovasculares ou morte, apesar da redução da incidência de nefropatia diabética. Em contraste, o tratamento intensivo mais rigoroso com o objetivo de reduzir a $\mathrm{HbAlc}$ abaixo de 6\% em pacientes com DM2, como ocorreu na Ação de Controle de Risco Cardiovascular no Diabetes (ACCORD), ${ }^{20}$ que não mostrou nenhum benefício na redução de eventos e resultou em um aumento da mortalidade, ganho de peso e risco de hipoglicemia. Os pacientes desse estudo apresentavam um elevado perfil de risco cardiovascular quando iniciado o tratamento intensivo do controle glicêmico. A tabela 1 apresenta as principais diferenças entre esses estudos.

Em pacientes com DM1, The Diabetes Control and Complications Trial (DCCT) $)^{21}$ mostrou os benefícios cardiovasculares do controle intensivo da glicemia após um acompanhamento de 17 anos. Os pacientes do DCCT foram tratados intensivamente durante 6,5 anos e acompanhados por 10 anos observacionalmente. Mesmo após o término do DCCT, o grupo intensivamente tratado apresentou uma redução de eventos cardiovasculares de $42 \%$.

A principal lição aprendida a partir desses resultados é que o tratamento intensivo da hiperglicemia, visando níveis de Alc menor que $7 \%$, quando iniciado precocemente em pacientes com curta duração de diabetes e de baixo risco cardiovascular, resulta em benefícios cardiovasculares. O mesmo não se aplica em

Tabela 1. Diferença dos efeitos do controle glicêmico na redução do risco cardiovascular em DM2

\begin{tabular}{|c|c|c|c|c|}
\hline & UKPDS-10 anos & VADT & ADVANCE & ACCORD \\
\hline Tamanho da amostra & $5.102 *$ & 1.791 & 11.140 & 10.251 \\
\hline Acompanhamento (anos) & 10 & 5,6 & 5 & 3,4 \\
\hline \multicolumn{5}{|l|}{ Características básicas: } \\
\hline Idade (anos) & 54 & 60,4 & 58 & 62,2 \\
\hline Duração do diabetes (anos) & Diagnóstico recente & 11,5 & 8 & 10 \\
\hline Presença de doença cardiovascular & $9 \%$ & $40 \%$ & $32 \%$ & $35 \%$ \\
\hline $\begin{array}{l}\text { Presença de complicações } \\
\text { microvasculares }\end{array}$ & $18 \%$ & $62 \%$ & $10 \%$ & $\begin{array}{c}\text { Albuminúria } \\
14.0(6,9-45,8)^{* *}\end{array}$ \\
\hline Níveis de A1c & $6,2 \%$ & $8,3 \%$ & $7,5 \%$ & $8,3 \%$ \\
\hline \multicolumn{5}{|l|}{ Efeitos do tratamento intensivo: } \\
\hline $\begin{array}{l}\text { Diferença nos níveis de A1c (intensivo/ } \\
\text { convencional) }\end{array}$ & $7,0 / 7,9 \%$ & $6,9 / 8,4 \%$ & $6,5 / 7,3 \%$ & $6,4 / 7,5 \%$ \\
\hline Redução em eventos macrovasculares & $\begin{array}{c}\text { Sulfa/grupo insulina: } \\
\downarrow 15 \% \mathrm{MI}, \downarrow 13 \% \text { death } \\
\text { Grupo metformina: } \\
\downarrow 33 \% \mathrm{MI}, \downarrow 27 \% \text { death }\end{array}$ & NS & NS & $\begin{array}{c}\downarrow \text { MI não fatal } \\
\text { 个óbito }\end{array}$ \\
\hline $\begin{array}{l}\text { Redução em eventos microvasculares } \\
\text { (retinopatia diabética, nefropatia ou } \\
\text { neuropatia) }\end{array}$ & $\begin{array}{c}\downarrow 24 \% \\
\text { (combinado) }\end{array}$ & NS & $\begin{array}{c}\downarrow \text { Incidência de } \\
\text { nefropatia }\end{array}$ & - \\
\hline
\end{tabular}

* 3.277 monitorização pós-pesquisa.

** A porcentagem dos indivíduos com complicações microvasculares não estavam disponíveis. Proporção de albumina urinária (mg) de creatinina (g); mediana (intervalo interquartil); NS, estatisticamente insignificante; A1c, hemoglobina glicada; MI, infarto do miocárdio. 
pacientes mais velhos expostos a hiperglicemia durante anos e com um perfil de maior risco cardiovascular. Nesses pacientes as metas glicêmicas devem ser menos rígidas.

Essa proteção inicial é resultado de um mecanismo conhecido como "memória metabólica", que significa que o efeito anterior da exposição à glicemia é recordado mais tarde, nos órgãos-alvo, ${ }^{15}$ resultando em efeitos deletérios ou protetores a longo prazo. Os mecanismos envolvidos nesse processo parecem compreender alterações epigenéticas e alterações metabólicas intracelulares que resultam do estresse oxidativo, inflamação suclínica e disfunção endotelial.

Diante desses resultados, a ADA em conjunto com a Associação Americana do Coração (AHA) e a Fundação do Colégio Americano de Cardiologia (ACCF) definiram as seguintes metas para o controle da glicemia em pacientes com diabetes: ${ }^{9}$

Tabela 2. Metas de controle glicêmico

\begin{tabular}{ll}
\hline HbA1c & \multicolumn{1}{c}{ Público } \\
\hline$<7,0 \%$ & - Todos \\
\hline & $\begin{array}{l}\text { - Pacientes que apresentem } \\
\text { pouca hipoglicemia }\end{array}$ \\
& - Curta duração de doença \\
& - Grande expectativa de vida \\
\hline & - Hipoglicemia recorrente \\
& - Pequena expectativa de vida \\
& - Complicações micro e \\
& macrovasculares avançadas
\end{tabular}

Fonte: Adaptado de Standards of Medical Care in Diabetes 2013 - American Diabetes Association.

\section{A importância das comorbidades}

\section{Obesidade}

A prevalência da obesidade está aumentando mundialmente e se tornando um grande problema de saúde pública devido à sua associação com doenças crônicas, como diabetes mellitus, hipertensão arterial, dislipidemia, apneia do sono, doença osteoarticular e cardio e cerebrovasculares.

De acordo com dados da OMS, em 2008 a prevalência global de obesidade (índice de massa corporal - IMC - $\geq 30 \mathrm{~kg} / \mathrm{m}^{2}$ ) foi de $10 \%$ em homens e $14 \%$ nas mulheres. Dados da National Health and Nutrition Examination Survey (NHANES) mostraram que a prevalência de sobrepeso e obesidade em adultos aumentou de $55,9 \%$ para $64,5 \%$ e de $22,9 \%$ para $30,5 \%$, de 1988-1994 para 1999-2000, respectivamente. ${ }^{22}$

A obesidade, principalmente a deposição de gordura visceral, está associada com inflamação de baixo grau, que tem um papel fundamental na patogênese do diabetes e em ambas as doenças está relacionado com aumento da morbidade e mortalidade devido à DCV.

Os principais determinantes para o aparecimento de diabetes estão além de fatores genéticos, são a obesidade e o sedentarismo. ${ }^{1}$ Vários estudos têm mostrado diminuição da incidência de diabetes por meio de tratamentos não farmacológicos, isto é, mudança de estilo de vida e redução do peso corporal. O estudo do Grupo de Prevenção do Diabetes mostrou que a incidência de diabetes foi reduzida em $58 \%$ no grupo que foi tratado com mudança intensiva do estilo de vida e permaneceu reduzida em 34\% após 10 anos de seguimento. ${ }^{23}$ Portanto, os esforços devem ser feitos para incentivar a adoção de estilo de vida saudável e, assim, combater a epidemia da obesidade.

\section{Dislipidemia}

A dislipidemia no DM2 piora o risco cardiovascular devido ao perfil aterogênico composto por aumento de lipoproteína de muito baixa densidade (VLDL), triglicerídeos e lipoproteína de baixa densidade LDL de colesterol e diminuição da lipoproteína de alta densidade (HDL) colesterol. Essas lipoproteínas, quando modificadas por oxidação e glicosilação, reduzem a complacência vascular predispondo a aterosclerose precoce e agressiva. ${ }^{22}$ Isso também pode ocorrer no DM1, apesar de serem pacientes jovens e anormalidades lipídicas raramente presentes, mas neste(s) caso(s) o perfil aterogênico não é causado apenas por níveis lipídicos aumentados, a hiperglicemia é também essencial nesse processo. ${ }^{21}$ Em muitos estudos 
de intervenção, a redução de colesterol LDL e triglicerídeos e o aumento do colesterol HDL provaram ser eficazes na redução da doença macrovascular e na mortalidade em pacientes com DM2, especialmente em pessoas com DCV anterior.

O Collaborative Atorvastatin on Diabetes Study (CARDS) foi o primeiro ensaio que analisou pacientes com DM2 sem DCV anterior. A intervenção com $10 \mathrm{mg}$ de atorvastatina mostrou redução de $37 \%$ em eventos cardiovasculares e redução de $48 \%$ em AVC quando comparada ao placebo. ${ }^{24}$ No estudo do tratamento de HDL atherosclerosis (HATS), o uso combinado de doses baixas de sinvastatina (10$20 \mathrm{mg} / \mathrm{dia}$ ) com doses elevadas de niacina (2-4 g/dia) mostrou uma redução no risco absoluto de $13 \%$ para DCV quando os níveis de HDL atingiram o alvo. ${ }^{25}$

O Treating to New Targets (TNT) estudou pacientes DM2 com DCV prévia e comparou o uso de atorvastatina $10 \mathrm{mg}$ (grupo convencional) com atorvastatina $80 \mathrm{mg}$ (grupo intensivo). A meta agressiva de LDL alcançada nesse estudo (73 mg/dl) apresentou as taxas mais reduzidas de mortalidade por eventos cardiovasculares entre todos os estudos com estatinas. ${ }^{26}$

Embora a diminuição do colesterol LDL tenha trazido evidências suficientes na redução da mortalidade cardiovascular no DM2, se o tratamento da dislipidemia começa muito tarde pode não ser eficaz para evitar a progressão da aterosclerose. De acordo com o Deutsche Diabetes Dialyze Study (4D), que estudou 1.255 pacientes com DM2 em estágio final da doença renal, randomizados para atorvastatina $20 \mathrm{mg} /$ dia ou placebo durante 4 anos, não houve redução significativa de eventos cardiovasculares com a intervenção quando comparado com placebo.

Em relação às metas do colesterol para os diabéticos, deve-se começar com os menores níveis possíveis de colesterol, como afirmado pelo National Cholesterol Education Program Adult Treatment Panel III Diretrizes (NCEP ATP III). ${ }^{22}$ Está bem estabelecido que os in- divíduos diabéticos são considerados como pertencentes a uma categoria de alto risco, portanto os benefícios da terapia na redução de LDL aparecem quando a meta de LDL-C de $70 \mathrm{mg} / \mathrm{dl}$ é alcançada. ${ }^{27}$

Em um recente ensaio, que analisou 22 estudos com estatinas versus controle, foi demonstrado que o uso de estatinas pode ser associado a um aumento da incidência de diabetes. ${ }^{27}$ Entretanto esse efeito foi mais de 50 vezes menor que o benefício absoluto observado com a terapia com estatina. Considerando hipertrigliceridemia, há pouca evidência para ratificar os benefícios quando as metas são alcançadas. Os fibratos são recomendados para reduzir o risco de pancreatite em pacientes com níveis de triglicerídeos acima de $400 \mathrm{mg} /$ $\mathrm{dl}$ quando a mudança do estilo de vida não tem sucesso. ${ }^{9}$ Até recentemente não havia dados que suportassem que o uso combinado de estatinas e fibratos podem reduzir a mortalidade cardiovascular. O The Fenofibrate Intervention and Event Lowering in Diabetes (FIELD) foi um estudo multinacional randomizado realizado com 9.795 pacientes com DM2, que mostrou que fenofibrato não reduziu significativamente o risco de eventos primários coronarianos. Em vez disso, ele reduziu o número total de eventos cardiovasculares (menos infartos do miocárdio não fatais e revascularização). ${ }^{28}$ Outra mensagem desse estudo foi que o fibrato confere possível proteção microvascular, porque reduziu a necessidade de tratamento a laser para retinopatia diabética.

\section{Hipertensão arterial sistêmica}

A HAS é uma doença altamente prevalente em todo o mundo e muito comum entre os pacientes com diabetes. Aproximadamente $10 \mathrm{a}$ $30 \%$ de diabéticos do tipo 1 e $60 \%$ dos doentes com DM2 têm hipertensão. ${ }^{9}$

A coexistência dessas duas condições aumenta o risco de desenvolvimento de complicações macrovasculares (IAM, AVC) e também complicações microvasculares (nefropatia e retinopatia). Dados obtidos dos certificados 
de óbito de pacientes diabéticos nos Estados Unidos da América estimam que cerca de 35\% a 75\% dos eventos cardiovasculares e complicações renais em pacientes diabéticos devem ser atribuídos à HAS coexistente. ${ }^{29}$

O início da HAS difere em diferentes tipos de diabetes. Em pacientes com DM1, a hipertensão se desenvolve anos após o diagnóstico, geralmente já refletindo o desenvolvimento da nefropatia diabética. A pressão arterial (PA) tende a aumentar 3 anos após o aparecimento de microalbuminúria. ${ }^{9}$ Em pacientes com DM2 a hipertensão pode estar presente no momento do diagnóstico, ou mesmo antes da elevação dos níveis de glicose no sangue. ${ }^{9}$ A associação entre a hipertensão e a obesidade está bem estabelecida levando a uma maior taxa de morbidade e mortalidade cardiovascular em pacientes com essas duas condições.

Estudos epidemiológicos mostraram que PA acima de 115/75 mmHg está associada a maior morbidade e mortalidade em indivíduos diabéticos. Eles também demonstraram que PA sistólica acima de $120 \mathrm{mmHg}$ está associada a maior evolução para doença renal terminal. Diversos ensaios clínicos controlados randomizados demonstraram redução de eventos cardiovasculares com a redução da PA sistólica para níveis inferiores a $140 \mathrm{mmHg}$ e da PA diastólica para níveis inferiores a $80 \mathrm{mmHg}$.9,30

O estudo ACCORD-BP avaliou o tratamento mais intensivo da pressão arterial (redução da PA sistólica com o objetivo de alcançar níveis inferiores a $120 \mathrm{mmHg}$ ) em pacientes com DM2 e DCV ou pelo menos 2 fatores de risco cardiovasculares e não mostrou redução nas taxas de eventos cardiovasculares (IAM, insuficiência cardíaca e morte cardiovascular). Foi observada uma redução no número de acidentes vasculares cerebrais com um número necessário para tratar de 89 e um aumento na incidência de efeitos colaterais. ${ }^{32}$

A PA-alvo recomendada para os doentes com diabetes, de acordo com a $\mathrm{ADA}^{9}$ é caracterizada por PA menor que $140 / 80 \mathrm{mmHg}$. Níveis inferiores a $130 / 80 \mathrm{mmHg}$ devem ser considerados em pacientes mais jovens com nefropatia diabética ou com risco aumentado de acidente vascular encefálico. ${ }^{9}$ Deve-se sempre considerar a individualização do tratamento, a resposta terapêutica e a tolerância às drogas. $\mathrm{O}$ tratamento da HAS em pacientes diabéticos visa a prevenção de DCV, a progressão da doença renal e da retinopatia diabética. $\mathrm{O}$ tratamento inicial deve incluir medidas não farmacológicas, como a redução de peso (em sobrepeso e obesidade), exercício regular, redução da ingestão de sal (< $1500 \mathrm{mg}$ por dia), do consumo de álcool (não mais do que duas porções por dia em homens e não mais do que uma porção por dia nas mulheres) e cessação do tabagismo. Terapia farmacológica deve ser iniciada em todos os diabéticos que persistem com PA maior que 140/80 mmHg, ou quando uma mudança no estilo de vida já foi implementado por 3 meses ou quando os níveis máximos da PA já são mais altos que 140/90 $\mathrm{mmHg}$ no diagnóstico. ${ }^{9,30,31}$

A terapia farmacológica pode ser realizada com várias classes de agentes anti-hipertensivos. Diuréticos, inibidores da enzima conversora da angiotensina, antagonistas da angiotensina II, betabloqueadores, bloqueadores do canal de cálcio, alfabloqueadores e a combinação de bloqueadores do sistema renina-angiotensina têm demonstrado ser eficazes na redução de eventos cardiovasculares. Na maioria dos casos, a associação de 2 ou 3 medicamentos pode ser necessária, a fim de alcançar os objetivos do tratamento. As tabelas 2 e 3 apresentam as metas de controle glicêmico, PA e lípides propostas pela ADA.

\section{Outros alvos de intervenção}

\section{Tabagismo}

O tabagismo é um importante fator de risco cardiovascular. Poucos estudos avaliaram seu papel de forma independente no subgrupo de pacientes diabéticos. Um estudo publicado em 2011 demonstrou que a interrupção do tabagismo em pacientes com DM2 estava associada à melhora dos parâmetros metabólicos, redução 
da PA e da albuminúria em 1 ano. ${ }^{33}$

A interrupção do tabagismo deve ser recomendada a todos os pacientes portadores de diabetes. $\mathrm{O}$ aconselhamento breve tem se mostrado eficaz e custo-efetivo para reduzir o consumo de tabaco entre pacientes tabagistas.

\section{Uso de fármacos antiagregantes}

A aspirina é um fármaco eficaz na prevenção secundária de eventos cardiovasculares em pacientes com alto risco cardiovascular. ${ }^{34}$ Diversos estudos foram realizados para avaliar a eficácia do uso da aspirina na prevenção primária de DCV em diversos grupos de pacientes. Dois estudos publicados em 2008 não foram capazes de mostrar uma redução significativa de eventos cardiovasculares em pacientes em uso de aspirina para prevenção primária.

Uma metanálise do Antithrombotic Trialists Collaborators reuniu 6 estudos com mais de 95.000 pacientes, sendo 4.000 diabéticos. $\mathrm{Na}$ análise final a aspirina mostrou uma redução do risco de eventos da ordem de $12 \%$. O maior impacto foi na redução de infarto não fatal com pequeno impacto na mortalidade cardiovascular e acidente vascular encefálico. ${ }^{34}$

Em 2010 a ADA publicou um posicionamento em conjunto com a American Heart Association e a American College of Cardiology Foundation, no qual foi recomendado o uso de aspirina em baixa dose (75-162 mg) para prevenção primária de DCV em pacientes diabéticos com risco de eventos cardiovasculares acima de $10 \%$ em 10 anos. ${ }^{35} \mathrm{O}$ uso de aspirina

\section{Tabela 3. Alvos terapêuticos no tratamento do} diabetes mellitus

\begin{tabular}{cc}
\hline Colesterol $(\mathrm{mg} / \mathrm{dl})$ & \\
Total & $<200$ \\
$\mathrm{HDL}$ & $>45$ \\
LDL & $<100$ \\
\hline Triglicerídeos $(\mathrm{mg} / \mathrm{dl})$ & $<150$ \\
\hline Pressão arterial $(\mathrm{mmHg})$ & $<140$ \\
Sistólica & $<80$ \\
Diastólica & $20-25$ \\
\hline Índice de massa corporal $\left(\mathrm{kg} / \mathrm{m}^{2}\right)$ & \\
\hline
\end{tabular}

Fonte: Adaptado de Standards of Medical Care in Diabetes 2013 - American Diabetes Association. não deve ser recomendado para paciente com baixo risco de eventos cardiovasculares $(<5 \%$ em 10 anos).

O clopidogrel também mostrou-se efetivo em reduzir eventos coronarianos em pacientes diabéticos, ${ }^{36}$ sendo indicado como adjuvante no primeiro ano após uma síndrome coronariana aguda e de forma isolada em pacientes intolerantes a aspirina.

\section{Perspectivas e conclusões}

O aumento global da prevalência das doenças crônicas, como obesidade, diabetes, hipertensão arterial e DCV, representa um importante desafio para a medicina interna. Considerando as dificuldades de atingirmos os alvos clínicos e metabólicos preconizados, o custo direto e indireto dessas doenças será cada vez maior e representará um importante ônus para a sociedade.

\section{Referências}

1. Wild S, Roglic G, Green A, Sicree R, King H. Global prevalence of diabetes: estimates for the year 2000 and projections for 2030. Diabetes Care. 2004;27(5):1047-53.

2. International Diabetes Federation. Diabetes Atlas. 3rd ed. Brussels, Belgium: International Diabetes Federation; 2006.

3. Negrato CA, Dias JP, Teixeira MF, Dias A, Salgado MH, Lauris JR, et al. Temporal trends in incidence of Type 1 diabetes between 1986 and 2006 in Brazil. J Endocrinol Invest. 2010;33:373-7.

4. American Diabetes Association. Economic costs of diabetes in the US in 2007. Diabetes Care 2008;31:596-615.

5. Orasanu G, Plutzky P. The pathologic continuum of diabetic vascular disease. J Am Coll Cardiol 2009 Feb 3;53(5 Suppl):S35-42. http://dx.doi. org:/10.1016/j.jacc.2008.09.055

6. Haffner SM, Lehto S, Rönnemaa T, Pyörälä $\mathrm{K}$, Laakso M. Mortality from coronary heart disease in subjects with type 2 diabetes and in nondiabetic subjects with and without prior myocardial infarction. $\mathrm{N}$ Engl J Med. 1998;339:229-34.

7. National Cholesterol Education Program (NCEP) Expert Panel on Detection, Evaluation, and Treatment of High Blood Cholesterol in Adults (Adult Treatment Panel III). Third Report of the National Cholesterol Education Program (NCEP) Expert Panel on Detection. Circulation 2002;106:3143-421. 
8. Bulugahapitiya U, Siyambalapitiya S, Sithole J, Idris I. Is diabetes a coronary risk equivalent? Systematic review and meta-analysis. Diabet Med 2009 Feb;26(2):142-8. Review. http://dx.doi. org:/10.1111/j.1464-5491.2008.02640.x

9. American Diabetes Association. Standards of medical care for patients with diabetes mellitus. Diabetes Care 2013:26(1): 533-50.

10. Stratton IM, Adler AL, Neil AW, Matthews DR, Manley SE, Cull CA, et al. Association of glycaemia with macrovascular and microvascular complications of type 2 diabetes (UKPDS 35): prospective observational study. BMJ 2000 Aug 12;321(7258):405-12.

11. The Diabetes Control and Complications Trial Research Group. The Effect of Intensive Treatment of Diabetes on the Development and Progression of Long-Term Complications in Insulin-Dependent Diabetes Mellitus. N Engl J Med 1993;329:977-86. http://dx.doi. org:/10.1056/NEJM199309303291401

12. Gomes MB, Gianella D, Faria M , Tambascia M, Fonseca RM, Réa R, et al. Prevalence of patients with diabetes type 2 within the targets of care guidelines in daily clinical practice: A multicenter study of type 2 diabetes in Brazil. Rev Diabet Stud. 2006 Summer;3(2):82-7.

13. Gomes MB, Coral M, Cobas RA, Dib SA, Canani LH, Nery M, et al. Prevalence of adults with type 1 diabetes who meet the goals of care in daily clinical practice: A nationwide multicenter study in Brazil Diabetes. Diabetes Res Clin Pract 2012 Jul; 97 (1): 63-70. http://dx.doi. org:/10.1016/j.diabres.2012.02.008

14. Matheus AS, Tannus LR, Cobas RA, Palma CC, Negrato CA, Gomes Mde B. Impact of Diabetes on Cardiovascular Disease: An Update. Int J Hypertens. 2013; 653-789. http://dx.doi. org:/10.1155/2013/653789

15. El-Osta A, Brasacchio D, Yao D, Pocai A, Jones PL, Roeder RG, et al. Transient high glucose causes persistent epigenetic changes and altered gene expression during subsequent normoglycemia. J Exp Med 2008 Sep 29;205(10):2409-17. http://dx.doi. org:/10.1084/jem.20081188

16. Gomes MB, Matheus ASM, Tibiriçá E. Evaluation of microvascular endothelial function in patients with type 1 diabetes using laser-Doppler perfusion monitoring: which method to choose? Microvasc Res 2008 Aug;76(2):132-3. http://dx.doi.org:/10.1016/j. mvr.2008.04.003

17. Holman RR, Paul SK, Bethel MA, Matthews DR, Neil HA. 10-Year Follow-up of Intensive Glucose Control in Type 2 Diabetes. N Engl J Med. 2008 Oct 9;359(15):1577-89. http://dx.doi. org:/10.1056/NEJMoa0806470.

18. Duckworth W, Abraira C, Moritz T, Reda D, Emanuele N, Reaven PD, et al. Glucose Control and Vascular Complications in Veterans with Type 2 Diabetes. N Engl J Med 2009 Jan 8;360(2):129-39. http://dx.doi.org:/10.1056/ NEJMoa0808431
19. ADVANCE Collaborative Group, Patel A, MacMahon S, Chalmers J, Neal B, Billot L, et al. Intensive Blood Glucose Control and Vascular Outcomes in Patients with Type 2 Diabetes. N Engl J Med. 2008 Jun 12;358(24):2560-72. http:// dx.doi.org:/10.1056/NEJMoa0802987

20. Action to Control Cardiovascular Risk in Diabetes Study Group, Gerstein HC, Miller ME, Byington RP, Goff DC Jr, Bigger JT, et al. Effects of Intensive Glucose Lowering in Type 2 Diabetes. N Engl J Med 2008 Jun 12;358(24):2545-59. http://dx.doi.org:/10.1056/ NEJMoa0802743

21. Nathan DM, Cleary PA, Backlund JY, Genuth SM, Lachin JM, The Diabetes Control and Complications Trial/Epidemiology of Diabetes Interventions and Complications (DCCT/EDIC) Study Research Group. Intensive Diabetes Treatment and Cardiovascular Disease in Patients with Type 1 Diabetes. N Engl J Med 2005 Dec 22;353(25):2643-53.

22. Grun Grundy SM, Cleeman JI, Merz CN, Brewer HB Jr, Clark LT, National Heart, Lung, and Blood Institute; American College of Cardiology Foundation; American Heart Association Implications of Recent Clinical Trials for the National Cholesterol Education Program Adult Treatment Panel III guidelines. Circulation 2004 Jul 13;110(2):227-39. Review.

23. Diabetes Prevention Program Research Group, Knowler WC, Fowler SE, Hamman RF, Christophi CA, Hoffman HJ, et al. 10-year follow-up of diabetes incidence and weight loss in the Diabetes Prevention Program Outcomes Study. Lancet. 2009 Nov 14;374(9702):1677-86. http://dx.doi.org:/10.1016/S0140-6736(09)614574

24. Colhoun HM, Betteridge DJ, Durrington PN, Hitman GA, Neil HA, Livingstone SJ,et al. CARDS investigators. Primary prevention of cardiovascular disease with atorvastatin in type 2 diabetes in the Collaborative Atorvastatin Diabetes Study (CARDS): multicentre randomised placebo-controlled trial. Lancet 2004 Aug 21-27;364(9435):685-96.

25. Brown BG, Zhao XQ, Chait A, Fisher LD, Cheung MC, Morse JS, et al.Sinvastatin and niacin, antioxidant vitamins, or the combination for the prevention of coronary disease. N Engl J Med. 2001 Nov 29;345(22):1583-92.

26. La Rosa JC, Grundy SM, Waters DD, Shear C, Barter P, Treating to New Targets (TNT) Investigators, et al. Intensive Lipid lowering with atorvastatin in patients with stable coronary disease. N Engl J Med 2005 Apr 7; 352(14):1425-35.

27. Cholesterol Treatment Trialists' (CTT) Collaborators, Mihaylova B, Emberson J, Blackwell L, Keech A, Simes J, et al. The effects of lowering LDL cholesterol with statin therapy in people at low risk of vascular disease: metaanalysis of individual data from 27 randomised trials. Lancet 2012 Aug 11;380(9841):581-90. http://dx.doi.org:/10.1016/S0140-6736(12)60367-5

28. Keech A, Simes RJ, Barter P, Best J, Scott R, FIELD 
study investigators, et al. Effects of long-term fenofibrate therapy on cardiovascular events in 9795 people with type 2 diabetes mellitus (the FIELD study): randomised controlled trial. Lancet. 2005 Nov 26;366(9500):1849-61.

29. Sowers JR, Esptein M. Diabetes Mellitus and Associated Hypertension, Vascular Disease, and Nephropathy: an Update. Hypertension. 1995 Dec;26(6 Pt 1):869-79. Review.

30. Chobanian AV, Bakris GL, Black HR, Cushman WC, Green LA, Izzo JL Jr, National Heart, Lung, and Blood Institute Joint National Committee on Prevention, Detection, Evaluation, and Treatment of High Blood Pressure; National High Blood Pressure Education Program Coordinating Committee. The Seventh Report of the Joint National Committee on Prevention, Detection, Evaluation, and Treatment of High Blood Pressure: the JNC 7 report. JAMA. 2003 May 21;289(19):2560-72.

31. Mancia G, Laurent S, Agabiti-Rosei E, Ambrosioni E, Burnier M, Caulfield MJ, European Society of Hypertension, et al. Reappraisal of European guidelines on hypertension management: a European Society of Hypertension Task Force document. J Hypertens. 2009 Nov;27(11):2121-58. http:// dx.doi.org:/10.1097/HJH.0b013e328333146d

32. ACCORD Study Group, Cushman WC, Evans GW, Byington RP, Goff DC Jr, Grimm RH Jr, et al. Effects of Intensive Blood-Pressure Control in Type 2 DiabetesMellitus. N Engl J Med. 2010 Apr 29;362(17):1575-85. http://dx.doi. org:/10.1056/NEJMoa1001286

33. Voulgari C, Katsilambros N, Tentolouris N. Smoking cessation predicts amelioration of microalbuminuria in newly diagnosed type 2 diabetes mellitus: a 1-year prospective study. Metabolism. 2011 Oct;60(10):1456-64. http:// dx.doi.org:/10.1016/j.metabol.2011.02.014

34. Antithrombotic Trialists' (ATT) Collaboration, Baigent C, Blackwell L, Collins R, Emberson J, Godwin J, et al. Aspirin in the primary and secondary prevention of vascular disease: collaborative meta-analysis of individual participant data from randomised trials. Lancet. 2009 May 30;373(9678):1849-60. http://dx.doi. org:/10.1016/S0140-6736(09)60503-1

35. PignoneM, Alberts MJ, Colwell JA, American Diabetes Association; American Heart Association; American College of Cardiology Foundation. Aspirin for primary prevention of cardiovascular events in people with diabetes: a position statement of the American Diabetes Association, a scientific statement of the American Heart Association, and an expert consensus document of the American College of Cardiology Foundation. Diabetes Care. 2010 Jun; 33(6):1395-402. http://dx.doi.org:/10.2337/ dc10-0555

36. Bhatt DL, Marso SP, Hirsch AT, Ringleb PA, Hacke W, Topol EJ. Amplified benefit of clopidogrel versus aspirin in patients with diabetes mellitus. Am J Cardiol. 2002 Sep 15;90(6):625-8. 